\title{
Determination of indium content of GaAs/(In,Ga)As/(GaAs) core-shell(-shell) nanowires by $x$-ray diffraction and nano x-ray fluorescence
}

\author{
Ali Al Hassan, ${ }^{1}$ R. B. Lewis, ${ }^{2}$ H. Küpers, ${ }^{2}$ W.-H. Lin, ${ }^{2}$ D. Bahrami, ${ }^{1}$ T. Krause, ${ }^{2}$ D. Salomon, ${ }^{3}$ \\ A. Tahraoui, ${ }^{2}$ M. Hanke, ${ }^{2}$ L. Geelhaar, ${ }^{2}$ and U. Pietsch ${ }^{1}$ \\ ${ }^{1}$ Naturwissenschaftlich-Technische Fakultät der Universität Siegen, 57068 Siegen, Germany \\ ${ }^{2}$ Paul-Drude-Institut für Festkörperelektronik, Leibniz-Institut im Forschungsverbund Berlin e.V., Hausvogteiplatz 5-7, 10117 Berlin, Germany \\ ${ }^{3}$ European Synchrotron Radiation Facility, 71 Avenue des Martyrs, 38000 Grenoble, France
}

(Received 16 September 2017; published 19 January 2018)

\begin{abstract}
We present two complementary approaches to investigate the In content in GaAs/(In,Ga)As/(GaAs) core-shell(shell) nanowire (NW) heterostructures using synchrotron radiation. The key advantage of our methodology is that NWs are characterized in their as-grown configuration, i.e., perpendicularly standing on a substrate. First, we determine the mean In content of the (In,Ga)As shell by high-resolution x-ray diffraction (XRD) from NW ensembles. In particular, we disentangle the influence of In content and shell thickness on XRD by measuring and analyzing two reflections with diffraction vector parallel and perpendicular to the growth axis, respectively. Second, we study the In distribution within individual NWs by nano x-ray fluorescence. Both the NW (111) basal plane, that is parallel to the surface of the substrate, and the $\{10-1\}$ sidewall plane were scanned with an incident nanobeam of $50 \mathrm{~nm}$ width. We investigate three samples with different nominal In content of the (In,Ga)As shell. In all samples, the average In content of the shell determined by XRD is in good agreement with the nominal value. For a nominal In content of $15 \%$, the In distribution is fairly uniform between all six sidewall facets. In contrast, in NWs with nominally $25 \%$ In content, different sidewall facets of the same NW exhibit different In contents. This effect is attributed to shadowing during growth by molecular beam epitaxy. At the same time, along the NW axis the In distribution is still fairly homogeneous. In NWs with $60 \%$ nominal In content and no outer GaAs shell, the In content varies significantly both between different sidewall facets and along the NW axis. This fluctuation is explained by the formation of (In, Ga)As mounds that grow simultaneously with a thinner (In, Ga)As shell. The methodology presented here may be applied also to other core-shell NWs with a ternary shell and paves the way to correlating NW structure with functional properties that depend on the as-grown configuration of the NWs.
\end{abstract}

DOI: 10.1103/PhysRevMaterials.2.014604

\section{INTRODUCTION}

The growth of semiconductor nanowires (NWs) has attracted significant interest in recent years because it enables the fabrication of novel semiconductor devices for future electronic and optoelectronic applications [1-4]. For example, NWs can absorb visible light more efficiently than planar structures due to the similarity between their size and the corresponding wavelength $[5,6]$. In addition, the formation of core-shell structures can further improve the performance of solar cells [7-11] and offers alternative opportunities for light emitting diodes [12-16]. In particular, due to their small diameter and high surface-to-volume ratio, core-shell NW heterostructures can be formed of highly lattice-mismatched materials allowing strain accommodation more efficiently than in the planar counterparts $[17,18]$. Furthermore, NW shells can provide sidewall passivation of the core material $[19,20]$, which can enhance the photoluminescence (PL) intensity $[8,21]$ and electron mobility [22]. Another advantage of NWs is the possibility to grow III-V semiconductor materials on Si substrates, which enables the integration of the good optical properties of direct band-gap materials with modern Si technology [23]. In this context, GaAs/(In,Ga)As core-shell NW heterostructures grown on $\mathrm{Si}$ are of particular interest, since they can be employed for applications based on near-infrared light such as optical data communication [12].

Naturally, the optical properties of such NW heterostructures depend on their structure, i.e., the chemical composition, the thicknesses of core and shell(s), the strain, and the crystal phase. Thus the precise characterization of these properties is indispensable for progress with respect to applications. We emphasize that variations between different NWs in an ensemble are common, and in particular the composition of ternary alloys may be inhomogeneous even within individual NWs. The most widely used methods for structural analysis at the nanoscale are based on transmission electron microscopy (TEM). In particular, compositional information can be deduced, e.g., from energy-dispersive x-ray spectrometry (EDX) and high-angle annular dark field (HAADF) images [24-28]. Alternatively, $\mathrm{x}$ rays may be used as a probe for structural characterization $[8,29]$. High-resolution $\mathrm{x}$-ray diffraction (XRD) reveals in specific directions periodicities of both the crystal lattice and heterostructures, which can be analyzed to deduce composition and thicknesses. Furthermore, $x$-ray fluorescence (XRF) directly provides chemical information. Traditionally, $\mathrm{x}$-ray based techniques do not exhibit high spatial resolution. This characteristic leads for NWs to the advantage that an ensemble average is obtained directly from the experiment, 
TABLE I. Structural information about the investigated samples. The NW density was extracted from SEM images, and the core-shell configuration, nominal dimensions, and nominal In content of the (In,Ga)As shell mentioned in the first four rows were deduced from MBE growth parameters. The In concentration $x$, thickness $t$, and the out-of-plane as well as in-plane elastic strain values of the (In,Ga)As shell listed in the last four rows were determined from XRD measurements along the [111] and [2-20] direction as described in the text. However, for sample 3 the In content had to be deduced from the parasitic growth on the substrate between the NWs.

\begin{tabular}{lccc}
\hline \hline & Sample 1 & Sample 2 & Sample 3 \\
\hline NW density $\left(\mu \mathrm{m}^{-2}\right)$ & 0.1 & 1 & 1 \\
Core-shell configuration & GaAs/(In,Ga)As/GaAs & GaAs/(In,Ga)As/GaAs & GaAs/(In,Ga)As \\
Nominal dimensions $(\mathrm{nm})$ & $140 / 40 / 30$ & $140 / 40 / 30$ & $140 / 18$ \\
Nominal $[\mathrm{In}](\%)$ & 15 & 25 & 60 \\
XRD [In] $(\%)$ & $15 \pm 0.7$ & $27 \pm 1.5$ & $70 \pm 8$ \\
XRD $[t](\mathrm{nm})$ & $40 \pm 2$ & $36 \pm 3$ & Not accessible \\
$\varepsilon_{z z, \mathrm{Ia}) \mathrm{As}}^{(\mathrm{In})}$ & -0.56 & -1.11 & Not accessible \\
$\varepsilon_{x x}^{(\mathrm{In}, \mathrm{Ga}) \mathrm{As}}(\%)$ & 0.93 & 1.59 & Not accessible \\
\hline \hline
\end{tabular}

while for TEM the sequential measurement of numerous NWs is required. At the same time, recent achievements in preparing $\mathrm{x}$-ray beams with nanometer diameter at synchrotron radiation sources [30] allow nowadays also the study of individual NWs [31-34]. The key benefit of the approach based on $\mathrm{x}$ rays is that NWs can be investigated in their as-grown configuration on the substrate, while characterization by TEM requires a destructive sample preparation. This benefit principally allows the correlation between structure and functional NW properties for one and the same NW that would be affected by sample preparation, such as PL, light coupling effects [35], or charge injection from the substrate [36].

In this paper, we report two complementary x-ray based methods for the structural analysis of core-shell NWs involving ternary compounds, exemplified by $\mathrm{GaAs} /(\mathrm{In}, \mathrm{Ga}) \mathrm{As} /(\mathrm{GaAs})$ core-shell-(shell) NW heterostructures. On the one hand, we employ high-resolution XRD in two orthogonal directions from NW ensembles in combination with scattering simulations based on a finite element method (FEM) approach to determine the average In content of the (In,Ga)As shell in the NW ensemble. In particular, the analysis of the full strain state along and perpendicular to the NW axis enables us to disentangle composition and thickness of the ternary shell. On the other hand, we use nano XRF measurements on single NWs to study the compositional homogeneity of the ternary shell.

\section{EXPERIMENTAL AND COMPUTATIONAL DETAILS}

The three investigated samples were grown by Ga-assisted molecular beam epitaxy (MBE) on $\mathrm{Si}$ (111) substrates. Details of the growth are described elsewhere [12,37]. For sample 1, a prepatterned substrate was used, resulting in an ordered array of NWs [38], while the other two NW ensembles are random. The NWs are typically $4 \mu \mathrm{m}$ long and composed of a 140-nm diameter $\mathrm{GaAs}$ core covered subsequently by (In, Ga)As and GaAs shells. The (In, Ga)As shells were deposited at a substrate temperature of about $405^{\circ} \mathrm{C}$ with high $\mathrm{As}_{2}$ : Ga flux ratio (atomic ratio above 40). The three different samples differ mostly in the nominal In content of the shells, which was varied by changing the $\mathrm{In} /(\mathrm{In}+\mathrm{Ga})$ flux ratio. A previous study of similar samples indicated that the crystal structure of these NWs is predominantly zinc blende with a small wurtzite content [37]. The shell thickness, $t$, and In shell content, $x$, of each sample under investigation are listed in Table I along with the NW number density and other information that will be introduced later.

Figure 1 shows exemplary scanning electron microscopy (SEM) images of single NWs from sample 2 [Fig. 1(a)] and sample 3 [Fig. 1(b)]. The NWs of samples 1 and 2 exhibit smooth shells whereas the morphology of NWs with high nominal In content on sample 3 is characterized by the presence of mounds resulting in a rough shell surface, as observed before [37] (overview SEM images of all three samples are shown in the Supplemental Material (SM) 1 [39]). In addition, both images display a dropletlike feature at the NW top. This feature results from the consumption of the Ga droplet at the NW top prior to the growth of the radial heterostructure and concurrent axial growth. Prior to the XRF measurements of selected individual NWs, approximately $2 \mu \mathrm{m}$ of the NW top were removed [sketch in Fig. 1(c)] using a focused ion beam (FIB), in order to simplify the characterization of the elemental distribution in the NW plane-view cross section. The dropletlike feature at the NW top exhibits in plane view a round shape [Fig. 1(d)], while the FIB procedure exposed the hexagonal cross section of the NW basal plane with the six well pronounced $\{110\}$ sidewall facets [Fig. 1(e)].
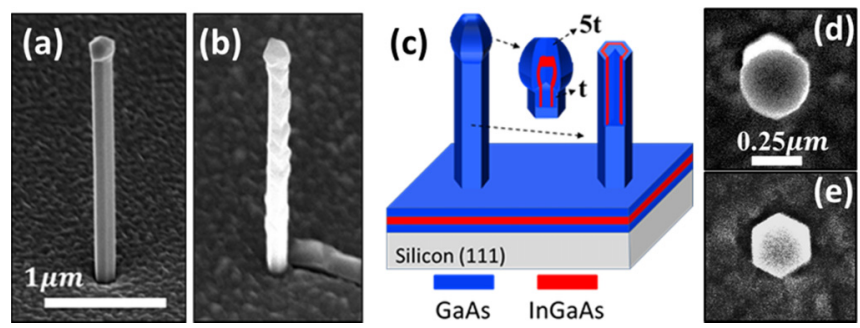

FIG. 1. SEM images of single GaAs/(In,Ga)As/(GaAs) core-shell NWs with (a) 25\% (sample 2) and (b) 60\% (sample 3) nominal In concentrations within the (In,Ga)As shell. The schematic in (c) illustrates the NW sample geometry including the NW top; " $t$ " represents the shell thickness on the sidewalls. During shell growth, deposition on the NW top proceeds with a rate five times faster compared to the shell growth rate due to the larger projected flux. As seen on the right-hand side, for the nano XRF measurement the NW top was removed by FIB. (d,e) Top-view SEM images of a single NW before (d) and after (e) FIB treatment, respectively. 


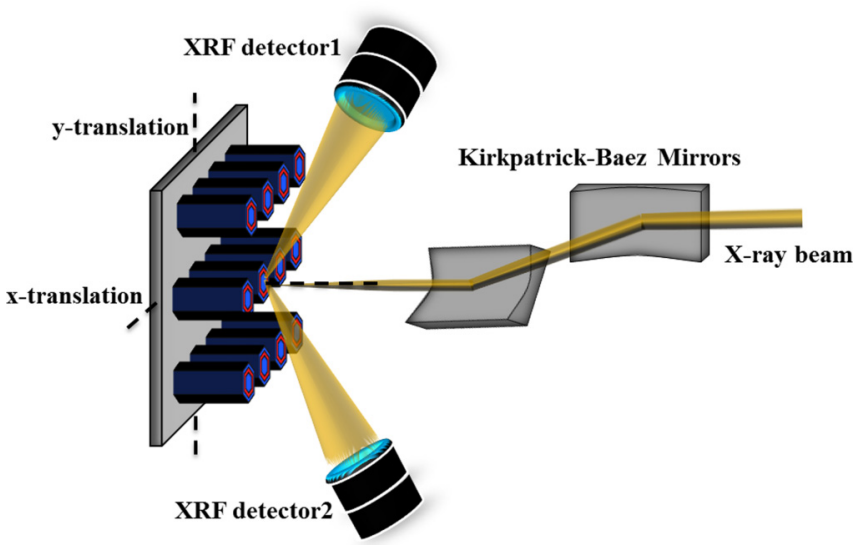

FIG. 2. A schematic illustration of the setup at the XRF station ID16b of the European Synchrotron Radiation Facility.

XRD measurements were carried out on NW ensembles at beamlines BL9 at the synchrotron DELTA (TU Dortmund) and P08 at PETRA III (DESY, Hamburg) using a photon energy of $13 \mathrm{keV}$, a beam size of $1 \times 1 \mathrm{~mm}^{2}$, and a two-dimensional (2D) detector. In particular, we recorded 2D reciprocal space maps (RSMs) in the vicinity of the axial (111) and the radial (2-20) Bragg reflections.

For scattering simulations, the strain field in a $\mathrm{GaAs} /(\mathrm{In}, \mathrm{Ga}) \mathrm{As}$ core-shell NW was computed using the numerical finite element method (FEM) approach. The FEM, which is based on linear elasticity theory, takes into account the hexagonal cross-sectional shape of the NW as well as the full anisotropy of the elastic constants. For the ternary alloy $(\mathrm{In}, \mathrm{Ga}) \mathrm{As}$, the elastic constants were obtained via linear interpolation of those of GaAs and InAs following Vegard's law. We employed the commercial software package MSC MARC $^{\oplus}$. The step width of the grid varied between 1 and $2 \mathrm{~nm}$ and was chosen such that the node density is higher in regions with strong strain gradients.

For the XRF study, we used the x-ray fluorescence nanoprobe station ID16B at the European Synchrotron Radiation Facility (Grenoble). Figure 2 shows a schematic view of the experimental setup. The nanoprobe is a white beam under normal incidence with upper energy cutoff at $29.46 \mathrm{keV}$, providing a photon flux density of $3.5 \times 10^{6} / \mathrm{s}$ in the nanobeam which was focused down to a spot size of $50 \times 50 \mathrm{~nm}^{2}$ [full width at half maximum (FWHM)] by a pair of dynamical Kirkpatrick-Baez mirrors. Due to its high photon energy, the nanobeam is transmitted through the whole nanostructure without major absorption loss, exciting element-specific fluorescence radiation. The fluorescence is recorded by two energy-dispersive silicon drift detectors placed at an angle of $15^{\circ}$ at opposing sides with respect to the sample. Illuminated by the probing beam, the sample is scanned with a step size of $10 \mathrm{~nm}$ along the NW basal plane for freestanding NWs or along the NW side plane of NWs lying on the substrate with an acquisition time of $0.5 \mathrm{~s}$ per step, which was sufficient to achieve highly intense $x$-ray fluorescence maps. Further details regarding the beamline instrumentation can be found elsewhere [40]. The elemental composition and local properties of the NWs were estimated by fitting the XRF spectra using the software PYMCA [41].

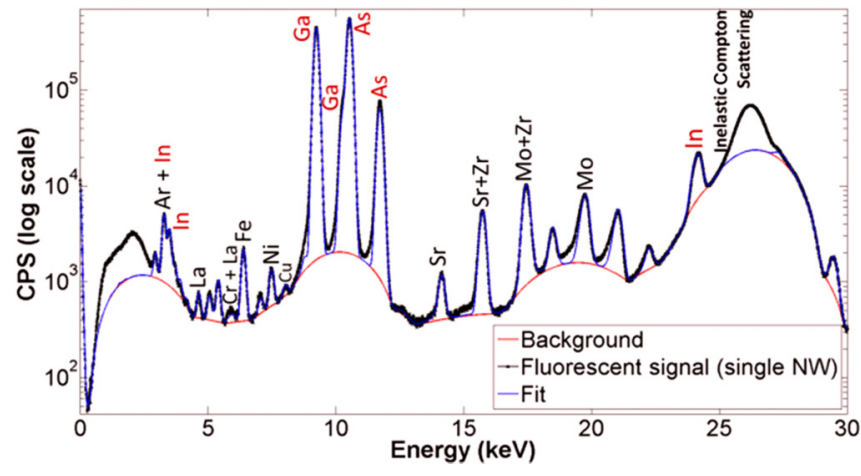

FIG. 3. Fluorescence energy spectrum acquired during the measurement of sample 1 . The lines are labeled with the elements that they correspond to. The spectrum contains lines originating from the sample (elements In, Ga, As marked in red) but also other lines originating from air and the experimental setup.

As the experiment is set under ambient conditions, the incident radiation interacts not only with the material inside the sample but also with air molecules and the material of the experimental hutch. The measured fluorescence energy spectrum of sample 1 after excitation by the incident $\mathrm{x}$-ray beam is shown in Fig. 3. The spectrum contains a large number of lines, out of which the ones associated with the elements In, $\mathrm{Ga}$, and As present in the sample are labeled in red. The other lines seen in the spectrum originate from material other than the investigated sample, e.g., the collimator, and are thus irrelevant.

\section{XRD AND FEM RESULTS AND DISCUSSION}

Due to the lattice mismatch between the GaAs core and the (In,Ga)As shell, the NW heterostructures are affected by epitaxial strain. Depending on the amount of lattice mismatch, this strain can relax plastically or accommodate elastically accompanied by lattice deformation. In a previous publication on such GaAs/(In,Ga)As core-shell NWs, Lewis et al. [37] did not observe any plastic strain relaxation up to an In content of $40 \%$. Thus in the present study we can safely analyze the results for samples 1 and 2 assuming entirely elastic relaxation. Furthermore, in the study by Lewis et al. [37], XRD profiles were acquired across the (111) reflection that reveals strain along the NW axis. In this direction, because of the pseudomorphic growth, the GaAs core and (In,Ga)As shell share the same lattice constant, whose value depends both on the In content in the shell and the dimensions of core and shell in the radial direction. Hence, for the deconvolution of shell thickness and In content, additional information is needed. Therefore, we measured for the present study RSMs both around the out-of-plane (111) and the in-plane (2-20) reflections (where the word "plane" refers to the substrate plane). As we will explain in more detail below, the combination of these two independent measurements allows the determination of the mean In content within the (In,Ga)As shell.

While the lattice constant along the growth direction (axial direction) is the same for core and shell materials, the lattice constant in the direction perpendicular to the growth axis (radial direction) varies within the core-shell heterostructure. 

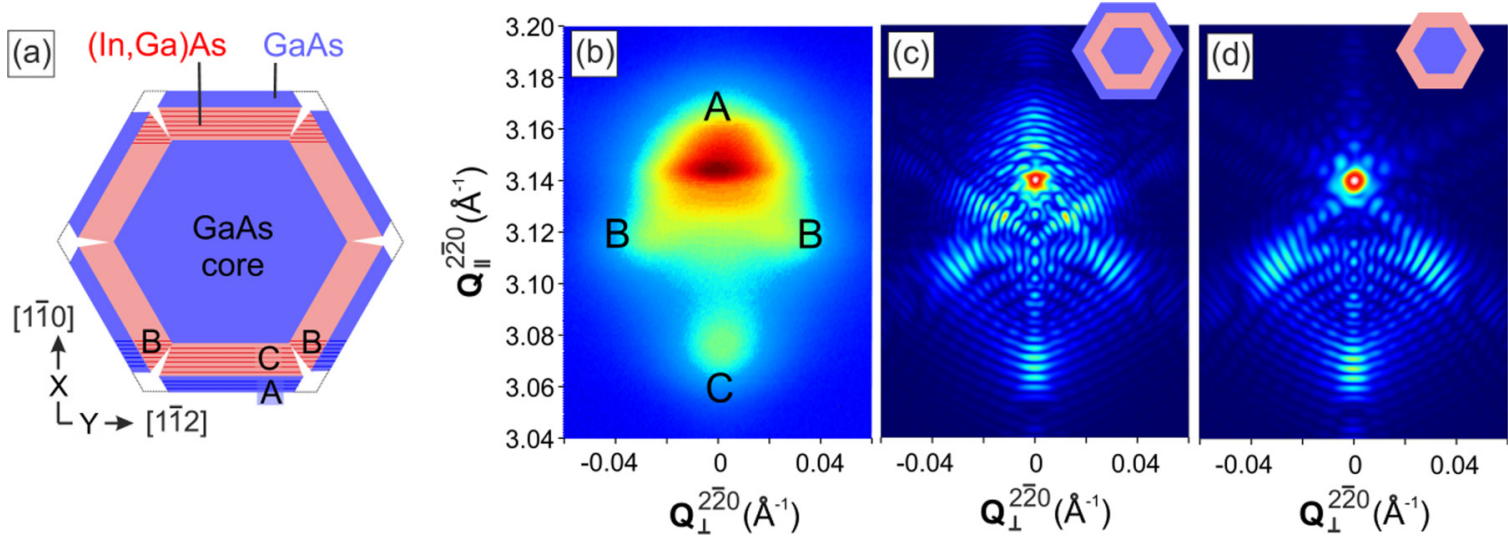

FIG. 4. Panel (a) illustrates the strain state along [2-20] for a single GaAs/(In,Ga)As/GaAs core-shell-shell NW heterostructure. Lines colored in red and blue and labeled "C" and "A" represent the (2-20) crystallographic planes of those (In,Ga)As and GaAs shell facets, respectively, that are oriented perpendicular to the direction scanned during out-of-plane XRD. Peak " $\mathrm{B}$ " corresponds to the (2-20) planes of the (In, Ga)As shell in neighboring facets. Note that these planes exhibit a different angle with respect to the facet orientation than the " $\mathrm{C}$ " planes, and thus different lattice spacing. The assignment is confirmed by finite element simulations presented in (c,d). As the strain state along the NW edges is expected to be different compared to the NW side facets, the areas close to the NW edges are kept empty in the schematic. (b) 2D RSM of the (2-20) reflection for sample 2. (c,d) show scattering simulations of the (2-20) reflection for a single $140 / 40 / 30 \mathrm{~nm} \mathrm{GaAs} /(\mathrm{In}, \mathrm{Ga}) \mathrm{As} / \mathrm{GaAs}$ core-shell-shell and 140/40 nm GaAs/(In,Ga)As core-shell NWs with nominal indium content of 25\%, respectively.

This variation is sketched in Fig. 4(a), which follows the model introduced by Stankevič et al. [42]. Analogous to planar films, it is supposed that the strain state within each of the six NW side facets is laterally homogeneous. As there may be deviations from this simplification at the edges of the hexagon, they are not included in the schematic. The red lines indicate (1-10) planes of the (In, Ga)As shell. The lattice spacing between these planes is different in sidewall facets that are oriented perpendicular to the [1-10] direction (label C) or inclined (label B). Both spacings differ, in turn, from the lattice spacing in this direction in the GaAs core. Furthermore, yet another value is expected for the outer GaAs shell (label A).

The variation in the in-plane-lattice constant becomes visible in the RSM taken at the (2-20) Bragg reflection of sample 2 displayed in Fig. 4(b). The two axes of this figure correspond to, on the one hand, the reciprocal vector $Q_{\|}^{2 \overline{2} 0}$ indicating the momentum transfer in the direction [2-20] measured in the XRD experiment and, on the other hand, the perpendicular reciprocal vector $\mathrm{Q}_{\perp}^{2 \overline{2} 0}$ [later on, similar notations are used for the (111) measurements]. The RSM contains, as expected, several peaks. The most intense one corresponds to the GaAs core. Compared to this peak, the strongest deviation along $\mathrm{Q}_{\|}^{2 \overline{2} 0}$ occurs in region $\mathrm{C}$ of the (In,Ga)As shell. In regions $\mathrm{B}$, the lattice planes of the $(\mathrm{In}, \mathrm{Ga}) \mathrm{As}$ shell are tilted by $\pm 60^{\circ}$ with respect to the direction of measurement, and the (1-10) lattice spacing lies in between the two extremes of the core and region $\mathrm{C}$. In other words, because the unit cells are orthorhombically deformed, the respective lattice parameters of these tilted planes are projections of the component of the (In,Ga)As shell. Depending on the inclination orientation of the sidewall facet, for region $\mathrm{B}$ momentum is additionally transferred either in the positive or negative direction $\mathrm{Q}_{\perp}^{2 \overline{2} 0}$, and correspondingly there are two symmetric peaks B in the RSM. For the outer GaAs shell, the change in (2-20) lattice spacing is compared to the GaAs core in the opposite direction as for the (In,Ga)As shell, and thus for region $\mathrm{A}$ a shoulder is visible on top of the core peak.
In order to substantiate the correlation between the different regions of the core-shell heterostructure and the different peaks in the RSM explained above, we simulated the diffraction pattern of the (2-20) reflection for a single NW model using kinematic scattering theory. The kinematic sum,

$$
I(q) \propto\left|\sum_{j} f_{\text {atom }, j}^{0} e^{i Q\left[r_{j}+u\left(r_{j}\right)\right]}\right|^{2},
$$

adds up the plane waves that are coherently scattered from each individual atom. Here, the atomic positions $r_{j}$ can be displaced from their ideal positions by a vector $u\left(r_{j}\right)$, e.g., due to strain, and $f_{\text {atom }, j}^{0}$ is the atomic scattering factor. The displacement of the atomic positions is obtained via interpolation of the ideal atomic positions with the three-dimensional (3D) displacement field of the core-shell (In,Ga)As/GaAs NW, simulated using a FEM approach. The result is presented in Fig. 4(c) and is in qualitative agreement with the experimental RSM in Fig. 4(b), thus confirming the assignment made above. Moreover, it is possible to retrieve the influence of the outer GaAs shell on the diffraction pattern by eliminating the respective nodes from the FEM model while maintaining the actual 3D displacement field of the full model. The result can be observed in Fig. 4(d). In this simulation, the peak A associated with the outer GaAs shell is not present.

To quantify the experimental XRD data, we created line profiles along $\mathrm{Q}_{\|}^{111}$ and $\mathrm{Q}_{\|}^{2 \overline{2} 0}$ from the RSMs of both the (111) and (2-20) reflections of all three samples, respectively, as shown in Figs. 5(a) and 5(b). This was done by integrating the intensity along $\mathrm{Q}_{\perp}^{111}$ and $\mathrm{Q}_{\perp}^{2 \overline{2} 0}$, respectively. Considering the (111) reflections in Fig. 5(a), the peak of the Si substrate, appearing at $\mathrm{Q}_{\|}^{111}=2.004 \dot{\mathrm{A}}^{-1}$, has the highest intensity and is used as a reference. Furthermore, there are three peaks related to the grown III-V materials. We associate the peak of the overall second highest intensity, labeled "PS," with the pseudomorphic lattice parameter of the core-shell NWs along 


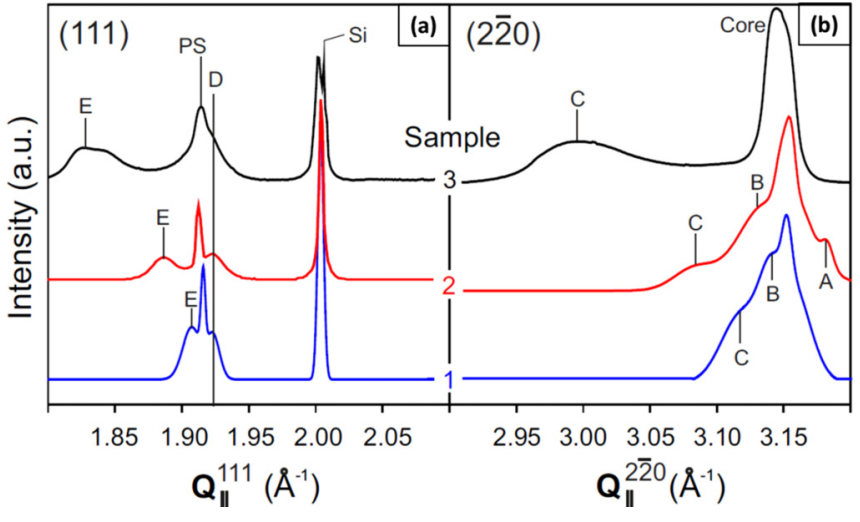

FIG. 5. (a,b) show XRD intensity integration of all samples plotted along scattering vectors $\mathrm{Q}_{\|}^{111}$ and $\mathrm{Q}_{\|}^{2 \overline{2} 0}$, respectively. For better visibility, the curves are plotted with vertical offsets. The position expected for unstrained $\mathrm{GaAs}$ in $\mathrm{Q}_{\|}^{111}$ in (a) is marked by the dashed line near "D." Positions expected for unstrained $\operatorname{In}_{0.15} \mathrm{Ga}_{0.85}$ As (blue), $\mathrm{In}_{0.27} \mathrm{Ga}_{0.73}$ As (red), and $\mathrm{In}_{0.7} \mathrm{Ga}_{0.3}$ As (black) are marked by vertical lines and named " $E$ " in (a) and " $C$ " in (b), respectively. A graphical explanation of peaks A-C is shown in Fig. 4(a). The explanation of the peaks named "A" through "E" and peak "PS" is given in the text.

the NW axis. In addition, the vertical lines labeled " $\mathrm{D}$ " and " $E$ " indicate the $Q_{\|}^{111}$ positions corresponding to relaxed cubic GaAs and (In,Ga)As with In contents close to the nominal values. Following the discussion in Ref. [37], these peaks are assumed to arise from parasitic growth on the substrate between the NWs. In particular, the vertical lines labeled "E" mark mean In contents of $15 \%, 27 \%$, and $70 \%$ for samples 1,2 , and 3, respectively. These values are in good agreement with the nominal values and are, for samples 1 and 2 , the result of an elaborate procedure to determine the In content in the (In,Ga)As shell from the XRD data that is described in the following.

First, the peaks in the line profiles were deconvoluted using a multi-Gaussian fit, and then the peak positions measured in $Q$ space were transformed into lattice parameters $d_{h k l}$. The relation between the reciprocal space coordinates $q_{h k l}$ and the lattice parameters in real space is given by

$$
\left|Q_{h k l}^{\text {expt }}\right|=2 \pi / d_{h k l}^{\text {expt }} .
$$

The lattice parameter can be calculated by

$d_{111}^{\mathrm{expt}}=d_{111}^{(\mathrm{In}, \mathrm{Ga}) \mathrm{As}} V+d_{111}^{\mathrm{GaAs}}(1-V)=d_{111}^{\mathrm{GaAs}}+\frac{0.404[\dot{\mathrm{A}}]}{\sqrt{3}} x V$

where $d_{111}^{(\mathrm{In}, \mathrm{Ga}) \mathrm{As}}$ and $d_{111}^{\mathrm{GaAs}}$ are the unstrained lattice parameters of (In,Ga)As and GaAs. " $x$ " represents the In content in the (In,Ga)As shell and " $V$ " is the volume fraction of the (In,Ga)As shell within the whole NW, which depends on the shell thickness " $t$ " [37]. For pseudomorphic growth, the (111) lattice spacing of the (In,Ga)As shell is compressed by $\varepsilon_{z z}^{(\mathrm{In}, \mathrm{Ga}) \mathrm{As}}$ with respect to $d_{111}^{(\mathrm{In}, \mathrm{Ga}) \mathrm{As}}$ whereas the GaAs core and outer shell parameters are tensile strained by $\varepsilon_{z z}^{\mathrm{GaAs}}$ with respect to $d_{111}^{\mathrm{GaAs}}$, expressed by

$$
d_{111}^{\mathrm{expt}}=d_{111}^{(\mathrm{In}, \mathrm{Ga}) \mathrm{As}}\left(1+\varepsilon_{z z}^{(\mathrm{In}, \mathrm{Ga}) \mathrm{As}}\right)=d_{111}^{\mathrm{GaAs}}\left(1+\varepsilon_{z z}^{\mathrm{GaAs}}\right) .
$$

Assuming the thicknesses of core and shells shown in Table I, the volume fraction of the (In,Ga)As shell is $V=0.367$ for all three samples. For the example of sample 2 with nominal In content $x=0.25$, one estimates $\varepsilon_{z z}^{(\mathrm{In}, \mathrm{Ga}) \mathrm{As}} \approx-0.011$ and $\varepsilon_{z z}^{\mathrm{GaAs}} \approx+0.0065$. However, as $d_{111}$ depends on the dimensions of the core and shells in addition to the In content, one cannot determine the In content in the (In, Ga)As shell from measuring the (111) reflection alone without knowing the core and shell thicknesses.

In Fig. 5(b) with the (2-20) measurements, a Si reference point is missing because the (2-20) peak of the Si substrate is not excited as the probing x-ray beam was nearly parallel to the surface of the substrate. Instead, the peak of highest intensity corresponds to unstrained GaAs. Thus we associate this peak with the lattice parameter of the GaAs core. Due to tensile strain along [111], the core is expected to be compressed along [2-20] by $\left(1+\varepsilon_{x x}^{\mathrm{GaAs}}\right)$. All other peak positions are measured with respect to this peak. In all samples, we find three additional peaks named $\mathrm{A}, \mathrm{B}$, and $\mathrm{C}$. In extension of the model introduced by Stankevič et al. for (In,Ga)N/GaN core- shell single NWs [42], we assign the center of the broad peak $\mathrm{C}$ to the (2-20) lattice parameter of the (In,Ga)As shell in sidewall facets parallel to (2-20), as explained above (Fig. 5). This lattice parameter will show an expansion $\left(1+\varepsilon_{x x}^{(\mathrm{In}, \mathrm{Ga}) \mathrm{As}}\right)$ due to the biaxial compressive strain along the [111] and [11-2] directions, $\varepsilon_{z z}$ and $\varepsilon_{y y}$, respectively. Following the notation in Ref. [43], the (2-20) lattice parameter can be expressed as

$$
\begin{aligned}
d_{2-20}^{(\mathrm{In}, \mathrm{Ga}) \mathrm{As}}(\mathrm{expt}) & =d_{2-20}^{(\mathrm{In}, \mathrm{Ga}) \mathrm{As}}\left(1+\varepsilon_{x x}^{(\mathrm{In}, \mathrm{Ga}) \mathrm{As}}\right) \\
& =d_{2-20}^{(\mathrm{In}, \mathrm{Ga}) \mathrm{As}}\left(1-v_{y x} \varepsilon_{y y}^{(\mathrm{In}, \mathrm{Ga}) \mathrm{As}}-v_{z x} \varepsilon_{z z}^{(\mathrm{In}, \mathrm{Ga}) \mathrm{As}}\right),
\end{aligned}
$$

using

$$
\varepsilon_{y y}^{(\mathrm{In}, \mathrm{Ga}) \mathrm{As}}=\frac{a^{(\mathrm{In}, \mathrm{Ga}) \mathrm{As}}-a_{(\mathrm{bulk})}^{\mathrm{GaAs}}}{a_{(\mathrm{bulk})}^{\mathrm{GaAs}}}
$$

and the directional Poisson ratios,

$$
\begin{aligned}
& v_{y x}=\frac{2\left(C_{11}+5 C_{12}-2 C_{44}\right)}{3\left(C_{11}+C_{12}+2 C_{44}\right)} \text { and } \\
& v_{z x}=\frac{4\left(C_{11}+2 C_{12}-2 C_{44}\right)}{3\left(C_{11}+C_{12}+2 C_{44}\right)},
\end{aligned}
$$

and

$$
d_{2-20}^{(\mathrm{In}, \mathrm{Ga}) \mathrm{As}}=\left(d_{2-20}^{\mathrm{GaAs}}+\frac{0.404[\dot{\mathrm{A}}]}{\sqrt{8}} x\right) .
$$

The tensile strain of the GaAs core along [111] will result in compressive strain along [11-2] and [110]. Assuming $\varepsilon_{y y}^{\mathrm{GaAs}}$ is given by the projection of the normal strain components of the (1-10) side planes tilted by $30^{\circ}$ with respect to the $y$ axis, $\varepsilon_{y y}^{\mathrm{GaAs}} \approx \frac{\sqrt{3}}{2} \varepsilon_{x x}^{\mathrm{GaAs}}$, which roughly follows the cylinder approximation $\varepsilon_{y y}^{\mathrm{GaAs}} \approx \varepsilon_{x x}^{\mathrm{GaAs}}$ [44], one may approximate

$$
\begin{aligned}
d_{2-20}^{\mathrm{GaAs}}(\text { expt }) & =d_{2-20}^{\mathrm{GaAs}}\left(1+\varepsilon_{x x}^{\mathrm{GaAs}}\right) \\
& \approx d_{2-20}^{\mathrm{GaAs}}\left(1-\left(\frac{v_{z x}}{1+\frac{v_{y x}}{2} \sqrt{3}}\right) \varepsilon_{z z}^{\mathrm{GaAs}}\right) .
\end{aligned}
$$


Here, $d_{2-20}^{\mathrm{GaAs}}$ and $d_{2-20}^{(\mathrm{In}, \mathrm{Ga}) \mathrm{As}}$ are the lattice parameters of the unstrained materials, $C_{i j}$ are the elastic constants [43,45], and the Poisson ratios are $v_{y x}=0.61, v_{z x}=0.49$. They are equally used for (In,Ga)As and GaAs.

Exemplarily the strain evaluation will be demonstrated for sample 2. Following Eq. (7), and considering $\varepsilon_{z z}^{\mathrm{GaAs}}$ taken from $d_{111}^{\text {expt }}$ using Eq. (4), the value $d_{2-20}^{\text {GaAs }}$ (expt) of the GaAs core is deformed by $1+\varepsilon_{x x}^{\mathrm{GaAs}}=1-0.654 v_{z x} \varepsilon_{z z}^{\mathrm{GaAs}} \approx 0.9980$ with respect to the unstrained value. In other words, the peak corresponding to the radially compressed GaAs core is found to be shifted by $\Delta q_{\|} \approx+0.004 \dot{\mathrm{A}}^{-1}$ towards larger values compared to $d_{2-20}^{\text {GaAs }}$. Following Eqs. (5) and (7) and using $\varepsilon_{x x}^{(\mathrm{In}, \mathrm{Ga}) \mathrm{As}}$ from Eq. (5), the value $d_{2-20}^{(\mathrm{In}, \mathrm{Ga}) \mathrm{As}}$ (expt) for (In,Ga)As is deformed by $1-v_{y x} \varepsilon_{y y}^{(\mathrm{In}, \mathrm{Ga}) \mathrm{As}}-v_{z x} \varepsilon_{z z}^{(\mathrm{In}, \mathrm{Ga}) \mathrm{As}} \approx 1.0164$ resulting in a peak shift of $\Delta q_{\|} \approx-0.031 \dot{\mathrm{A}}^{-1}$ compared to the unstrained value. Because Eq. (3) contains the product $x V$, but Eq. (5) depends on $x$ only, both $x$ (In content) and $V$ (i.e., shell thickness $t$ ) can be disentangled by measuring $d_{111}$ (expt) and $d_{2-20}^{(\mathrm{In}, \mathrm{Ga}) \mathrm{As}}(\mathrm{expt})$. Following this procedure, the mean In contents are estimated to be $x=0.150 \pm 0.007$ and $0.270 \pm 0.015$ with shell thicknesses of $40 \pm 2 \mathrm{~nm}$ and $36 \pm 3 \mathrm{~nm}$ for samples 1 and 2 , respectively, as shown in Table I. Using these strain values, the unit cell of the (In,Ga)As shell is uniaxially deformed by $1-\frac{1+\varepsilon_{x,}^{(\mathrm{In}, \mathrm{G}) \mathrm{As}}}{1+\varepsilon_{z 2}^{\mathrm{II}, \mathrm{G} a \mathrm{As}}} \approx 1 \%$ and $2 \%$, for samples 1 and 2 , respectively, towards the radial direction.

For sample 3 with a nominal In content of $60 \%$, according to the results of Lewis et al. [37] an additional path for strain relaxation beyond the elastic deformation regime is relevant. For such structures Lewis et al. demonstrated a scenario for strain relaxation, where a coherently strained (In,Ga)As NW shell and plastically relaxed (In,Ga)As mounds grow simultaneously on the GaAs NW sidewalls. Detailed calculations of such a complex relaxation mechanism are underway but beyond the scope of the present study. Therefore, we suppose peak $\mathrm{E}$ originates from parasitic islands which corresponds to unstrained ( $\mathrm{In}, \mathrm{Ga})$ As with mean indium content of $x=0.70 \pm 0.08$.

The main outcome of the XRD analysis is the mean In content of the (In,Ga)As shell in the NW ensembles of samples 1 and 2 listed in the fifth row of Table I. These values are in very good agreement with the nominal values. For sample 3, we measured the mean In content from parasitic islands and suppose that the same In content is incorporated in the NWs.

\section{XRF RESULTS AND DISCUSSION}

The XRD analysis of the previous section provides an ensemble average for the In content of the (In,Ga)As shell. However, in many cases it is crucial to obtain information about the In distribution within the shell, and for this purpose measurements of individual core-shell NWs are indispensable. In addition, nano XRF is the method of choice to measure single NWs in their as-grown configuration on the substrate.

In our experiments, the (111) plane of the samples was probed by a Gaussian x-ray beam with diameter of $50 \mathrm{~nm}$ (FWHM). The Gaussian beam shape results in an inhomogeneous fluorescence excitation within the probing area, as sketched in Fig. 6(a). At the marked position, the (In,Ga)As
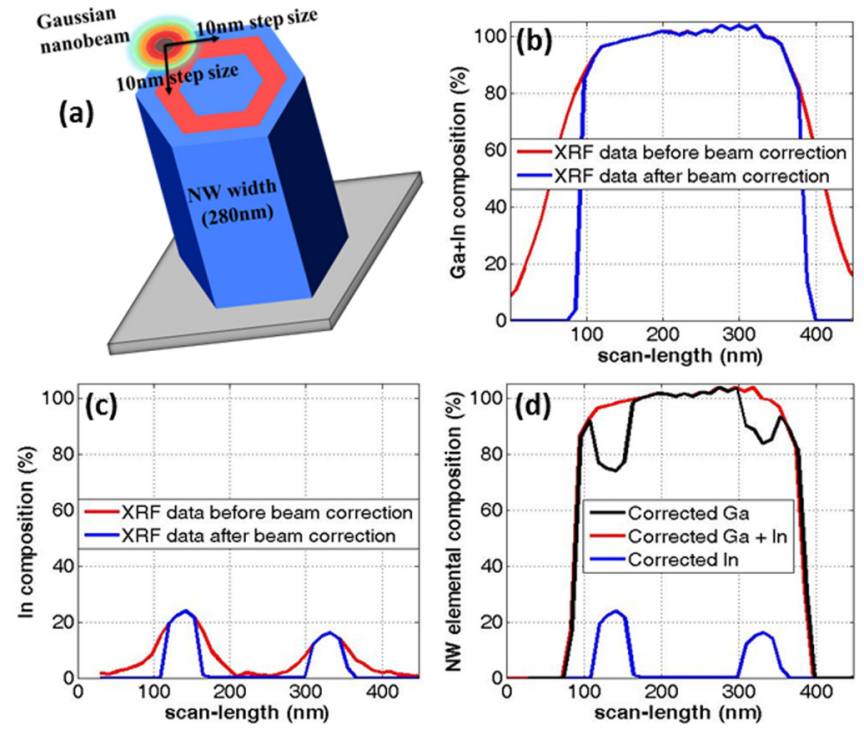

FIG. 6. (a) Sketch of how the X-ray nanobeam is scanned across the NW area during the XRF experiment. (b) Combined Ga and In fluorescence signal along single line scans through the center of the NW before (red) and after (blue) Gaussian beam shape correction. (c) In fluorescence signal along single line scans before (red) and after (blue) Gaussian beam shape correction. (d) Corrected Ga (black), In (blue), and sum of Ga and In (red) signals.

shell is excited by the center of the Gaussian beam, whereas the neighboring GaAs areas are excited by the tails of the beam. In order to determine the true elemental distribution, the fluorescence intensity map has to be corrected for the Gaussian beam profile. To this end, a simulation program was created using a MATLAB code that mimics the correct excitation profile at each probing position and normalizes the XRF intensity generated at a certain sample area to the Gaussian incident intensity distribution. The feasibility of this procedure was tested on sample 2, simulating the expected In distribution and assuming a Gaussian beam FWHM of $50 \mathrm{~nm} \times 50 \mathrm{~nm}$, the nominal NW diameter of $280 \mathrm{~nm}$, and an (In,Ga)As shell thickness of $40 \mathrm{~nm}$. The measured total Ga+In signal is shown in Fig. 6(b) as a red line and the corrected profile is shown in blue. The FWHM of the blue box reflects the nominal NW diameter very well. The same procedure was applied to the In signal within the (In,Ga)As shell, and the result is presented in Fig. 6(c). Here, the simulated intensity was normalized to unity and the beam corrected In profile is shown in blue. The Ga composition shown in Fig. 6(d) was then obtained by subtracting the corrected In signal in Fig. 6(c) from the total signal in Fig. 6(b), which reproduces the core-shell-shell composition of the NW. This procedure was applied in a similar way to determine the In and Ga profiles measured for sample 1.

The corrected 2D XRF intensity maps of the In signal averaged over the $K_{\alpha}, K_{\beta}$, and $L_{\alpha}$ fluorescence intensities taken from the (111) basal plane of individual NWs of samples 1-3 are shown in Fig. 7 in false color representation. For samples 1 and 2, each data point of the map is an average over the sample area excited by the nano $\mathrm{x}$-ray beam after correction for the Gaussian beam profile. For both samples, we assumed that the In content is homogeneous along the NW length and 

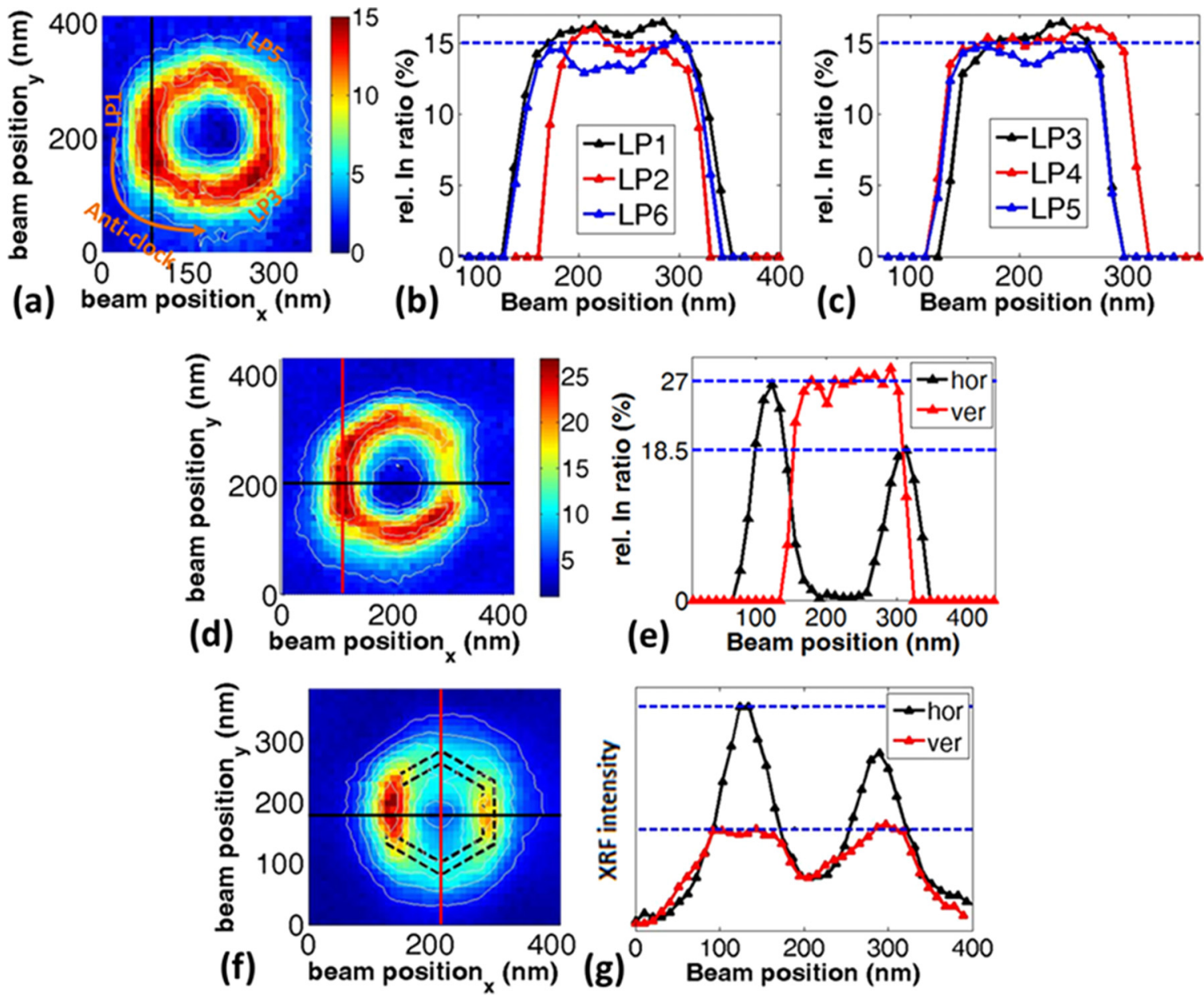

FIG. 7. (a) XRF intensity map of a single NW from sample 1 after correction for the Gaussian beam profile. The six sidewall facets are labeled counterclockwise by LP1-LP6. The black line indicates for LP1 the central position of a line profile that was extracted and is displayed in (b). For this line profile, the XRF intensity was integrated across the shell thickness. Similar line profiles were extracted for all six facets and are displayed in (b,c). (d) XRF intensity map of a single NW from sample 2 after correction for the Gaussian beam profile. The red and black line mark the positions of the line scans shown in (e), one parallel to a side facet (red) and one across the NW (black). (f) XRF intensity map of a single NW from sample 3. Due to the irregular morphology of the NWs on this sample, the signal could not be corrected for the Gaussian beam profile. The dashed lines represent the expected position of the (In,Ga)As shell. The solid lines indicate the positions of the line scans shown in (g), both across the NW, in the horizontal (black) and vertical (red) direction.

reaches both detectors mainly through the NW side planes with negligible absorption. For sample 1 [Fig. 7(a)] the measured In distribution of a selected NW reflects the hexagonal NW geometry with six well pronounced $\{110\}$ side facets. In order to probe the homogeneity of the In distribution within the shells, we extracted line scans along each of the six NW side planes marked as LP1-LP6 by integrating over the respective shell thicknesses, and the measured In fluorescence intensity was normalized to the mean In content of the shell determined from the XRD data (see Table I). The resulting line scans are shown in Figs. 7(b) and 7(c) and reveal a rather uniform In distribution. Differences in In content between the different side facets do not exceed $1.5 \%$ (in percentage points). The In distribution in other single NWs of the same sample shows qualitatively similar behavior (see Fig. S2.1 in the SM [39]).

Figure 7(d) displays the In fluorescence of one selected NW of sample 2. Here, the In signal is not uniformly distributed across the six side planes but shows a significantly lower In content along one of the side planes. In order to quantify these differences, we took line profiles along the $x$ and the $y$ directions across the NW basal plane [Fig. 7(e)]. The mean In signal of the profile extracted in the $y$ direction was normalized to the In content of $27 \%$ found by XRD (Table I). With this normalization, the line scan along the $x$ axis cutting a pair of opposite facets reveals maximum In concentrations of $27 \%$ and $18.5 \%$ at the left and right lobes, respectively [Fig. 7(e)]. However, we emphasize that the inhomogeneity in the measured In fluorescence can be explained both by a lower In content and/or a thinner (In,Ga)As shell thickness for the right facet compared to the left one. Because the probing nanobeam is larger than the shell thickness, these effects cannot be distinguished. Additional single NWs measured from this sample show qualitatively similar behavior (see Fig. S2.2 in the SM [39]).

A possible explanation for the differences in In fluorescence observed for the NWs of sample 2 on different sidewall facets is shadowing of the molecular In and Ga beams during shell growth. For a similar sample, TEM revealed for some NWs 

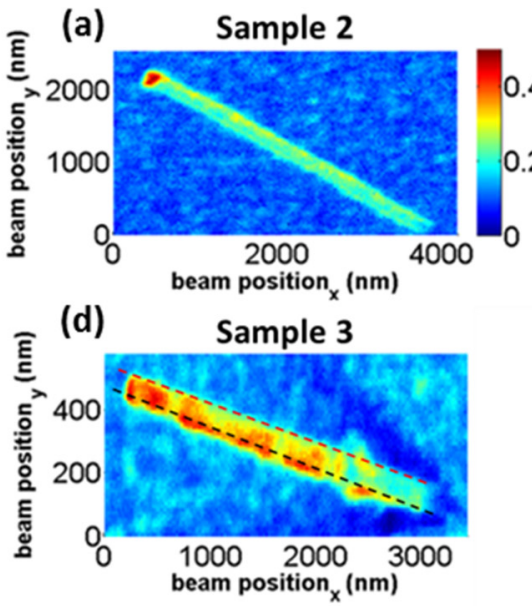

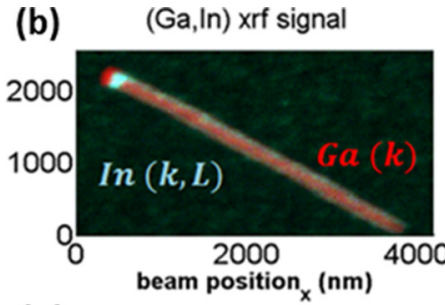

(e)

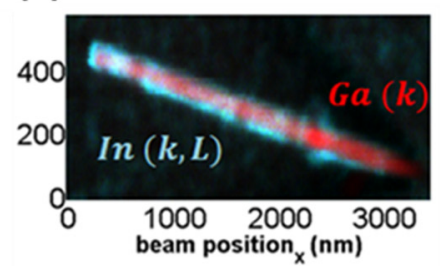

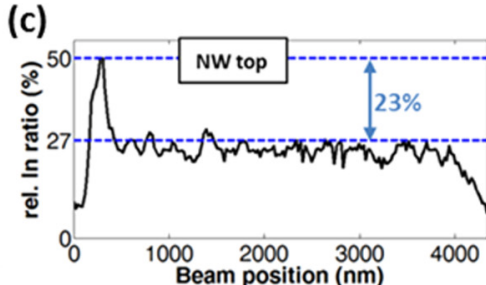

(f)

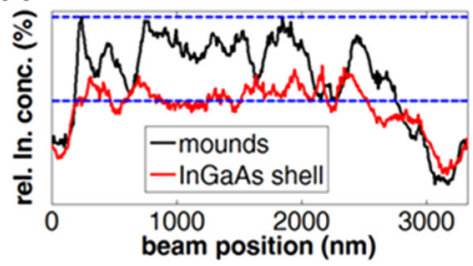

FIG. 8. XRF measurements of NWs oriented perpendicular to the probing beam. (a) In fluorescence map of a single NW from sample 2. (b) Overlaid map of the In (cyan) and Ga (red) fluorescence. (c) Line profile of the In content along the whole NW length, resulting from normalizing the In fluorescence signal to the average In content of the (In,Ga)As shell determined by XRD. Note that at the dropletlike feature at the NW tip (left-hand side), the probed volume of In is different, and that hence the quantitative value there cannot be directly compared. (d) In fluorescence map of a single NW from sample 3. (e) Overlaid map of the In (cyan) and Ga (red) fluorescence. (f) Line scans of the In fluorescence taken along the NW length through its center (red) and at the NW edge through the (In,Ga)As mounds (black).

a notably different (In,Ga)As shell thickness on few sidewall facets, in particular if another NW was close by [28]. We note that for the present study the NW number is higher for sample 2 than for sample 1, which would be consistent with more pronounced shadowing. Also, on sample 1 the spacing between the NWs is homogeneous due to the prepatterning, while for the unpatterned substrate of sample 2 the spacing varies.

A completely different picture is revealed for the NWs of sample 3 with nominally $60 \%$ In concentration in the (In, Ga)As shell. The 2D In distribution of one selected NW from sample 3 is shown in Fig. 7(f). The dotted lines show the expected shape of the (In,Ga)As shell, assuming the nominal NW structure with a GaAs core of $140 \mathrm{~nm}$ in diameter surrounded by $18 \mathrm{~nm}$ of (In,Ga)As shell. We find that the In fluorescence signal is not uniformly distributed. Instead, it shows strong maxima at certain positions along the shell and much lower intensity in other shell regions. Similar patterns were obtained for other NWs from the same sample (Fig. S2.3 in the SM [39]). Qualitatively, this behavior is visualized in Fig. 7(g) by line scans along the $x$ axis (black line) and $y$ axis (red line). Due to the nonuniform NW morphology characterized by the appearance of (In,Ga)As mounds [cf. Fig. 1(b)], the Gaussian beam shape correction of the XRF maps was not feasible for this sample, and therefore a determination of shell and mound thickness is not possible. Lewis et al. found that for NWs with the same core-shell dimensions and nominal In concentration, the 18-nm-thick (In,Ga)As shell splits into a 10-nm-thick coherently strained (In,Ga)As shell and thicker plastically relaxed (In,Ga)As mounds [37]. In our study, the larger thickness of the mounds compared to the shell results in a much higher XRF intensity. This effect would explain the differences observed for the scan along the $x$ axis in Fig. 7(g) compared to the line scan along the $y$ axis. We note that the higher intensity of the mounds compared to the shell does not automatically indicate a higher In content as one has to consider the different excitation volumes. Following the estimate presented in SM 3 [39], we cannot conclude from our
XRF experiment whether the In content differs in the mounds and in the coherent shell.

During the XRF experiment, we observed that some of the NWs were broken or had grown in an oblique direction with respect to the surface normal. These NWs offer the opportunity to investigate the In distribution along the NW axis. For such NWs, the XRF intensity was not corrected for the beam profile. Figure 8(a) shows the In fluorescence map of such a NW from sample 2. Since these NWs were not treated by FIB, they still display the dropletlike top at one end of the NW, and there the In fluorescence is clearly much higher. In the map of Fig. 8(b), both the In and Ga fluorescence are plotted, each in a different color. Apart from the dropletlike feature at the tip, the fluorescence is fairly homogeneous along the whole NW length. Quantitative information is provided by the line profile in Fig. 8(c), for which the In fluorescence was scaled to the average In content of $27 \%$ in the (In, Ga)As shell obtained by XRD. We note that the absolute In fluorescence intensity cannot be compared with the one measured for the vertical NWs since now much less material from the (In,Ga)As shell is probed for each pixel of the scan. At the dropletlike feature at the NW top, the In content is $23 \%$ (percentage points) higher than in the NW itself. This result demonstrates the importance of removing the NW tip to retrieve meaningful data for the vertical NWs. However, we would like to point out that on the basis of these data, the In content actually cannot be compared in a quantitative way between the tip and the rest of the NW, since the probed amount of In-containing material is different at the tip. Along the NW itself, the In content fluctuates by around $7 \%$ (percentage points). This fairly small variation justifies the averaging done along the $\mathrm{NW}$ axis for the XRF results presented in Fig. 7.

The situation is again different for the NWs of sample 3. Data analogous to the ones discussed in the previous paragraph are shown for a representative NW of sample 3 in Figs. 8(d)-8(f). In this case, the In fluorescence exhibits strong fluctuations along the NW length, to an extent that the 
dropletlike feature at the tip cannot be clearly distinguished. These local fluctuations of the XRF intensity reach up to $24 \%$ with respect to the maximum XRF intensity. These fluctuations are consistent with the fluctuations observed for the vertical NWs and are again explained by the formation of pronounced mounds on the NW sidewalls. These mounds lead to a locally larger volume of (In, Ga)As compared to the coherent shell. In an attempt to learn more about these mounds, we extract one line scan along the NW through its center [red line in Fig. 8(f)] and one line scan at the edge of the NW (black line), where the latter preferentially crosses the mounds. Not surprisingly, the relative fluctuations in In fluorescence are stronger for the line scan at the NW edge. However, when approximating the mounds by a box with depth equal to the width of a NW side plane, following the arguments given in SM 3 [39], we still cannot judge whether the In content differs in mounds and shell.

\section{SUMMARY}

We have presented two independent and complementary experimental studies of the In content in GaAs/(In,Ga)As core-shell NWs based on $x$ rays. First, by measuring both the axial (111) and radial (2-20) reflections of NW ensembles using $\mathrm{XRD}$, we were able to disentangle the average In concentration of the (In,Ga)As shell and its thickness despite the ternary nature of the alloy. The resulting values are in good agreement with the nominal ones deduced from growth parameters. Second, we demonstrated on individual NWs the measurement of the spatial In distribution in a 40-nm-thick (In,Ga)As shell by nano XRF, employing a Gaussian nanobeam with FWHM of $50 \mathrm{~nm}$. By correcting the measured XRF intensities for the Gaussian beam shape, we were able to identify In fluctuations within the (In,Ga)As shells. Furthermore, by normalizing the XRF signal to the average In content determined by XRD, we obtained quantitative information about the variation in In fluorescence.
NWs with $15 \%$ nominal In content in the shell exhibited a fairly homogeneous In distribution between different sidewall facets. In contrast, in core-shell NWs with $25 \%$ nominal In content in the shell, we observed significant variations between the In fluorescence on opposite sidewall facets of the same NW. However, we have to point out that this measurement method by itself cannot distinguish between differences in the In content at constant shell thickness and differences in shell thickness at constant In content, since the fluorescence intensity depends only on the total amount of probed In. For the same sample, we found in a different measurement geometry that the In content is fairly homogeneous along the length of the NW. Core-shell NWs with $60 \%$ nominal In content in the shell exhibit pronounced fluctuations in In fluorescence, both between different sidewall facets and along the length of the NW. These fluctuations are consistent with the formation of (In,Ga)As mounds on the sidewall facets during growth.

We emphasize that the measurement techniques presented here can be applied to NWs in their as-grown perpendicular configuration on the growth substrate. Thus these techniques pave the way to correlating at the single-NW level structural information and functional properties depending on the asgrown configuration of the NWs. Finally, these techniques can be transferred to core-shell NWs with a ternary shell in general.

\section{ACKNOWLEDGMENTS}

We are grateful to M. Matalla and O. Krüger at FerdinandBraun-Institut, Berlin, for substrate prepatterning. Also, we acknowledge experimental support by beamline scientists, $\mathrm{Ch}$. Sternemann from DELTA (TU Dortmund), and K. Perumal and F. Bertram from PETRA III (DESY). This work was supported by Deutsche Forschungsgemeinschaft, under Grants No. Pi217/38 and No. Ge2224/2. R.B.L. acknowledges additional funding from the Alexander von Humboldt Foundation.
[1] N. P. Dasgupta, J. Sun, C. Liu, S. Brittman, S. C. Andrews, J. Lim, H. Gao, R. Yan, and P. Yang, Adv. Mater. 26, 2137 (2014).

[2] R. Yan, D. Gargas, and P. Yang, Nat. Photonics 3, 569 (2009).

[3] Y. Zhang, J. Wu, M. Aagesen, and H. Liu, J. Phys. D: Appl. Phys. 48, 463001 (2015).

[4] Y. Li, F. Qian, J. Xiang, and C. M. Lieber, Mater. Today 9, 18 (2006).

[5] E. Garnett and P. Yang, Nano Lett. 10, 1082 (2010).

[6] M. D. Kelzenberg, W. S. Boettcher, J. Petykiewicz, D. B. Turner-Evans, M. C. Putnam, E. L. Warren, J. M. Spurgeon, R. M. Briggs, N. S. Lewis, and H. A. Atwater, Nat. Mater. 9, 239 (2010).

[7] E. Nakai, M. Yoshimura, K. Tomioka, and T. Fukui, Jpn. J. Appl. Phys. 52, 055002 (2013).

[8] J. Treu, M. Bormann, H. Schmeiduch, M. Döblinger, S. Morkötter, S. Matich, P. Wiecha, K. Saller, B. Mayer, M. Bichler, M. C. Amann, J. J. Finley, G. Abstreiter, and G. Koblmüller, Nano Lett. 13, 6070 (2013).
[9] P. Krogstrup, H. I. Jørgensen, M. Heiss, O. Demichel, J. V. Holm, M. Aagesen, J. Nygard, and A. Fontcuberta i Morral, Nat. Photonics 7, 306 (2013).

[10] K. Moratis, S. L. Tan, S. Germanis, C. Katsidis, M. Androulidaki, K. Tsagaraki, Z. Hatzopoulos, F. Donatini, J. Cibert, Y. M. Niquet, H. Mariette, and N. T. Pelekanos, Nano. Res. Lett. 11, 176 (2016).

[11] J. C. Shin, K. H. Kim, K. J. Yu, H. Hu, L. Yin, C-Z. Ning, J. A. Rogers, J.-M. Zuo, and X. Li, Nano Lett. 11, 4831 (2011).

[12] E. Dimakis, U. Jahn, M. Ramsteiner, A. Tahraoui, J. Grandal, X. Kong, O. Marquardt, A. Trampert, H. Riechert, and L. Geelhaar, Nano Lett. 14, 2604 (2014).

[13] X. Dai, A. Messanvi, H. Zhang, C. Durand, J. Eymery, C. Bougerol, F. H. Julien, and M. Tchernycheva, Nano Lett. 15, 6958 (2015).

[14] C. P. Svensson, T. Mårtensson, J. Trägårdh, C. Larsson, M. Rask, D. Hessman, L. Samuelson, and J. Ohlsson, Nanotechnology 19, 305201 (2008). 
[15] M. Tchernycheva, V. Neplokh, H. Zhang, P. Lavenus, L. Rigutti, F. Bayle, F. H. Julien, A. Babichev, G. Jacopin, L. Largeau, R. Ciechonski, G. Vescovig, and O. Kryliouk, Nanoscale 7, 11692 (2015).

[16] K. Tomioka, J. Motohisa, S. Hara, K. Hiruma, and T. Fukui, Nano Lett. 10, 1639 (2010).

[17] K. L. Kavanagh, Semicond. Sci. Technol. 25, 024006 (2010).

[18] K. L. Kavanagh, I. Saveliev, M. Blumin, G. Swadener, and H. E. Ruda, J. Appl. Phys. 111, 044301 (2012).

[19] O. Demichel, M. Heiss, J. Bleuse, H. Mariette, and A. Fontcuberta i Morral, Appl. Phys. Lett. 97, 201907 (2010).

[20] P. Parkinson, H. J. Joyce, Q. Gao, H. H. Tan, X. Zhang, J. Zou, C. Jagadish, L. M. Herz, and M. B. Johnston, Nano Lett. 9, 3349 (2009).

[21] C. C. Chang, C. Y. Chi, M. Yao, N. Huang, C. C. Chen, J. Theiss, A. W. Bushmaker, S. LaLumondiere, T.-W. Yeh, M. L. Povinelli, C. Zhou, P. D. Dapkus, and S. B. Cronin, Nano Lett. 12, 4484 (2012).

[22] J. W. W. Van Tilburg, R. E. Algra, W. G. G. Immink, M. Verheijen, E. P. A. M. Bakkers, and L. P. Kouwenhoven, Semicond. Sci. Technol. 25, 024011 (2010).

[23] T. Mårtensson, C. P. T. Svensson, B. A. Wacaser, M. W. Larsson, W. Seifert, K. Deppert, A. Gustafsson, L. R. Wallenberg, and L. Samuelson, Nano Lett. 4, 1987 (2004).

[24] S. Biswas, J. Doherty, D. Saladukha, Q. Ramasse, D. Majumdar, M. Upmanyu, A. Singha, T. Ochalski, M. A. Morris, and J. D. Holmes, Nat. Commun. 7, 11405 (2016).

[25] H. Potts, M. Friedl, F. Amaduzzi, K. Tang, G. Tütüncüoglu, F. Matteini, E. A. Lladó, P. C. McIntyre, and A. Fontcuberta i Morral, Nano Lett. 16, 637 (2016).

[26] K. Wei Ng, W. Son Ko, R. Chen, F. Lu, T.-T. D. Tran, K. Li, and C. Chang-Hasnain, J. ACS Appl. Mater. Interfaces 6, 16706 (2014).

[27] K. Tomioka, M. Yoshimura, and T. Fukui, Nature 488, 189 (2012).

[28] J. Grandal, M. Wu, X. Kong, M. Hanke, E. Dimakis, L. Geelhaar, H. Riechert, and A. Trampert, Appl. Phys. Lett. 105, 121602 (2014).

[29] M. Keplinger, Th. Mårtensson, J. Stangl, E. Wintersberger, B. Mandl, D. Kriegner, V. Holý, G. Bauer, K. Deppert, and L. Samuelson, Nano Lett. 9, 1877 (2009).
[30] G. E. Ice, J. D. Budai, and J. W. L. Pang, Science 334, 1234 (2011).

[31] J. Segura-Ruiz, G. Martínez-Criado, C. Denker, J. Malindretos, and A. Rizzi, Nano Lett. 14, 1300 (2014).

[32] G. Martínez-Criado, A. Homs, B. Alén, J. A. Sans, J. SeguraRuiz, A. Molina-Sánchez, J. Susini, J. Yoo, and G.-C. Yi, Nano Lett. 12, 5829 (2012).

[33] J. Wallentin, D. Jacobsson, M. Osterhoff, M. T. Borgström, and T. Salditt, Nano Lett. 17, 4143 (2017).

[34] D. Dzhigaev, T. Stankevič, Z. Bi, S. Lazarev, M. Rose, A. Shabalin, J. Reinhardt, A. Mikkelsen, L. Samuelson, G. Falkenberg, R. Feidenhans'1, and I. A. Vartanyants, ACS Nano 11, 6605 (2017).

[35] I. Giuntoni, L. Geelhaar, J. Bruns, and H. Riechert, Opt. Express 24, 18417 (2016).

[36] G. Bussone, H. Schäfer-Eberwein, E. Dimakis, A. Biermanns, D. Carbone, A. Tahraoui, L. Geelhaar, P. H. Bolívar, T. U Schülli, and U. Pietsch, Nano Lett. 15, 981 (2015).

[37] R. B. Lewis, L. Nicolai, H. Küpers, M. Ramsteiner, A. Trampert, and L. Geelhaar, Nano Lett. 17, 136 (2017).

[38] H. Küpers, A. Tahraoui, R. B. Lewis, S. Rauwerdink, M. Matalla, O. Krüger, F. Bastiman, H. Riechert, and L. Geelhaar, Semicond. Sci. Technol. 32, 115003 (2017).

[39] See Supplemental Material at http://link.aps.org/supplemental/ 10.1103/PhysRevMaterials.2.014604 for SEM images of all samples and further nano XRF and AFM analysis.

[40] G. Martínez-Criado, J. Villanova, R. Tucoulou, D. Salomon, J. P. Suuronen, S. Labouré, C. Guilloud, V. Valls, R. Barrett, E. Gagliardini, Y. Dabin, R. Baker, S. Bohic, C. Cohen, and J. Morse, J. Synchrotron Radiat. 23, 344 (2016).

[41] V. Solé, E. Papillon, M. Cotte, P. Walter, and J. Susini, Spectrochim. Acta, Part B 62, 63 (2007).

[42] T. Stankevič, S. Mickevičius, M. S. Nielsen, O. Kryliouk, R. Ciechonski, G. Vescovi, Z. Bi, A. Mikkelsen, L. Samuelson, C. Gundlach, and R. Feidenhans'l, J. Appl. Crystallogr. 48, 344 (2015).

[43] J. Grönqvist, N. Søndergaard, F. Boxberg, T. Guhr, S. Åberg, and H. Q. Xu, J. Appl. Phys. 106, 053508 (2009).

[44] O. Salehzadeh, K. L. Kavanagh, and S. P. Watkins, J. Appl. Phys. 114, 054301 (2013).

[45] www.ioffe.ru/sva/nsm/semicond/gaas/mechanic.html. 